\title{
THE MOBILE USER ENVIRONMENT: SMART PHONES, PORTABLE MEDIA PLAYERS (PMPS), AND TABLETS
}

\subsection{INTRODUCTION}

Mobile connectivity is becoming ubiquitous for voice, video, and data. A significant percentage of people now carry powerful smartphones and/or tablets that enable them to be productive, connected, entertained, and instrumented while on the move, away from their offices or homes. Clearly, there has been an evolution over time for people on the move, from being able to get "nothing" (up to mid-1980s), to being able to get voice (since the mid-1980s), to getting data (such as e-mails) (mostly from the mid1990s), to accessing applications (data and location-based applications) (mostly since the early 2000s), and now also to get real-time and/or streaming and/or on-demand video. According to recent Nielsen data, over 28 million people in the U.S. watched video content on their mobile phones in 2011 , with a large ( $>40$ percent) increase from 2010; monthly usage of video exceeds 4 hours, as documented in Appendix 1.1A.

The network fabric has transitioned from analog, to digital (time-division multiplexing), to packet technology, especially using voice over IP (VoIP) for voice and using IP version 4 (IPv4) for applications. However, in recent years, there has been a steady depletion of the pool of available IPv4 address blocks; a point of exhaustion was reached in 2011, when only 1 percent of the address space remained available. Service providers are now, of necessity, planning to give serious consideration to the imminent rollout of IP version 6 (IPv6) infrastructures, to parallel the existing IPv4 infrastructure, in order to maintain growth and provide customers with new enhanced services. Mobile IPv6 (MIPv6) is a version of IPv6 that intrinsically supports active,

Mobile Video with Mobile IPv6, First Edition. Daniel Minoli.

(C) 2012 John Wiley \& Sons, Inc. Published 2012 by John Wiley \& Sons, Inc. 
real-time device movement across a wide geography (it supports a concept similar to mobile IP in the version 4 world, but with added capabilities). MIPv6 allows mobile nodes (MNs) to maintain persistent IP connectivity while the MN moves around in an IPv6 network. It has been adopted in $3 \mathrm{G}$ code division multiple access (CDMA) networks for handling host-based mobility management, specifically as a way to maintain connectivity when the MN moves between access routers (ARs). In addition to the basic set of initial MIPv6 protocols, several enhancements have been added in the past few years.

At the same time there has been increased interest in new forms of IP-based video distribution, both in terms of the underlying streaming or IP television (IPTV) and/or content distribution networks (CDNs) technology, as well as in terms of the content providers and content creation itself. User-generated video (UGV), "for Web publishing" of original content, video on demand ( $\mathrm{VoD})$, and time-shifted video, are seeing steady market penetration. Consumers expect to be able to get access to such content not only on their standard or smart (connected) TV, but also on their smartphones, portable media players (PMPs), and tablets. MIPv6 offers an ideal opportunity to support the evolving consumer paradigm of mobility, productivity, connectivity, entertainment, and instrumentation. It follows that there is interest on the part of service providers to explore the technology, protocols, deployment strategies, and approaches to IPv6-based mobility in general, and IPv6 mobile video in particular. MIPv6 allows session (e.g., Transmission Control Protocol (TCP) session) continuity-while some video applications utilize User Datagram Protocol (UDP), other video applications do use TCP.

The types of content that people typically get with a mobile video device include entertainment (nonlinear video such as music videos, short clips from YouTube/Web $\mathrm{TV}$, and so on) and real-time information (linear video such as breaking news, emergency reports, weather, local/regional news, live events as they happen, and traffic) [1]. One should keep in mind that there are no substitutes for entertainment ${ }^{1}$ (one needs a video stream), but there are substitutes for news/weather/traffic information: one might simply look at the home page of CNN, FNC, TWC, and so on, to get that type of information via traditional Web browsing - video may or may not actually be required in all these instances.

Besides MIPv6, there are a number of ways to deliver video to a mobile device including but not limited to-European Telecommunications Standards Institute (ETSI) Digital Video Broadcast Handheld (DVB-H), International Telecommunication Union-Telecommunications (ITU-T) IPTV, Internet Engineering Task Force (IETF) IPv4, IETF Mobile IPv4 (MIP), IETF IPv6, Open Mobile Video Coalition (OMVC) mobile digital TV, and vendor-proprietary methods. Each of these methods has advantages and disadvantages. This investigation focuses mostly on MIPv6; we believe to be the first textbook on this topic. An overview of the approach and capabilities afforded by MIPv6 is provided in this introductory chapter. The chapters that follow expand in greater details the concepts introduced herewith.

\footnotetext{
${ }^{1}$ We exclude videogames in this discussion.
} 


\subsection{BASIC MIPv6 OPERATION}

For video distribution, as well as for other applications to smartphones and similar devices, there is a desire to support direct communication between MNs (also known as mobile hosts) and far-end destinations, whether such far ends are themselves a stationary node or another MN. Such far end destination could be, for example, a video streaming service provider. In order to efficiently maintain reachability, thus supporting flexible mobility, the goal is to retain the same explicit IP address regardless of the real-time location or specific network elements and/or networks used to support connectivity. This is not easily achievable with IPv4 for a number of reasons; however, MIPv6 described in Request for Comments (RFC) 3775, "Mobility Support in IPv6" (June 2004), among others ${ }^{2}$, facilitates this task. RFC 3775 is known as the "MIPv6 base specification." RFCs are specifications and related materials published by the IETF. IPv6 mobility, specifically MIPv6, relies on IPv6 capabilities.

RFC 3775 notes that without specific support for mobility in IPv6, packets destined to an MN would not be able to reach it while the MN is away from its home network. In order to continue communication in spite of its movement, an MN could change its IP address each time it moves to a new link, but the MN would then not be able to maintain transport and higher-layer connections when it changes location. Mobility support in IPv6 is particularly important, as mobile users are likely to account for a majority, or at least a substantial fraction, of the population of the Internet during the lifetime of IPv6. MIPv6 allows nodes to remain reachable while moving around in the IPv6 Internet: it enables a device to change its attachment point to the Internet without losing higher-layer functionality through the use of tunneling between it and a designated home agent (HA). Stated another way, MIPv6 enables an MN to maintain its connectivity to the Internet when moving from one AR to another, a process referred to as handover. Figure 1.1 depicts some of the elements involved in IPv6 mobility and their basic functionality.

IPv6 was originally defined in RFC 1883 but was then obsolete by RFC 2460 , "Internet Protocol, Version 6 (IPv6) Specification," authored by S. Deering and R. Hinden (December 1998). A large body of additional RFCs has emerged in recent years to add capabilities and refine the IPv6 concept. IPv6 embodies IPv4 best practices but removes unused or obsolete $\mathrm{IPv} 4$ characteristics; this results in a betteroptimized Internet protocol. Some of the advantages of IPv6 include the following:

- Scalability and expanded addressing capabilities: IPv6 has 128-bit addresses versus 32-bit IPv4 addresses. With IPv4, the theoretical number of available IP addresses is $2^{32} \sim 10^{10}$. IPv6 offers a much larger $2^{128}$ space. Hence, the number of available unique node addressees is $2^{128} \sim 10^{39}$. IPv6 has more than 340 undecillion $(340,282,366,920,938,463,463,374,607,431,768,211,456)$ addresses, grouped into blocks of 18 quintillion addresses.

${ }^{2}$ See Appendix 1B for a more inclusive listing of MIPv6 RFCs. 


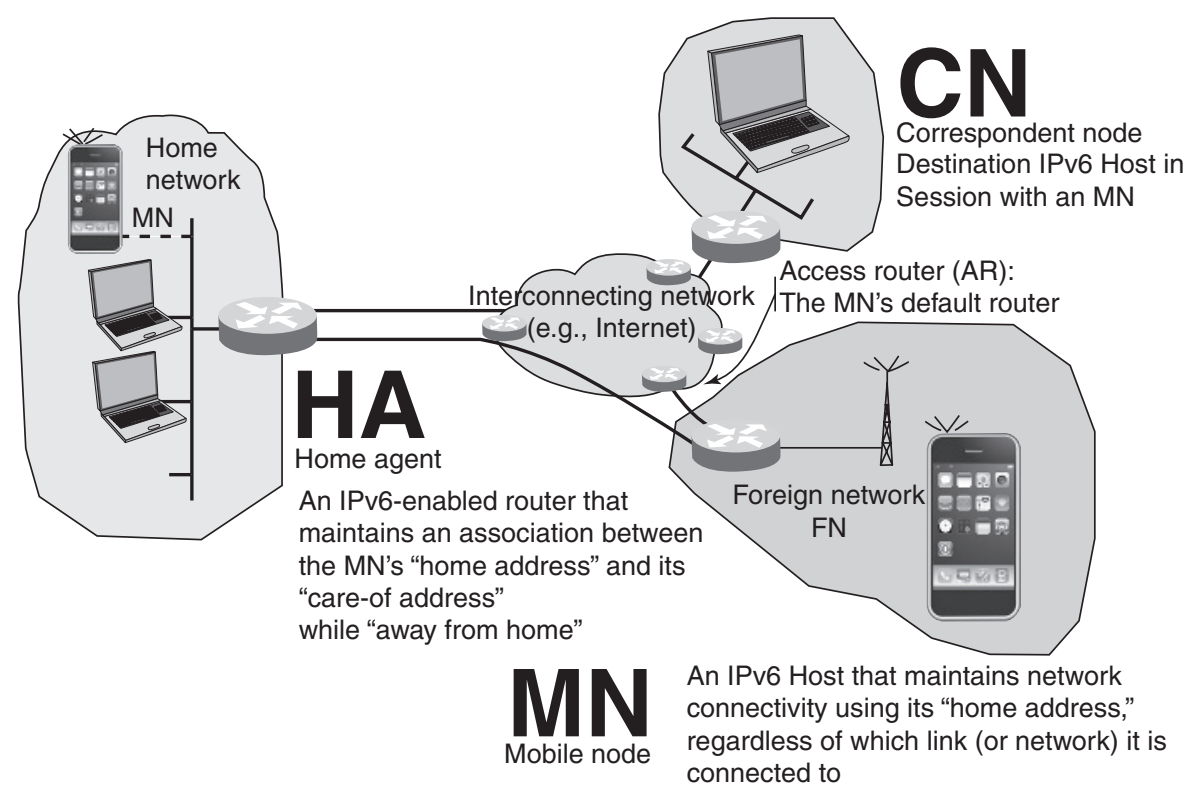

FIGURE 1.1 Basic MIPv6 Environment

- "Plug-and-play": IPv6 includes a "plug-and-play" mechanism that facilitates the connection of equipment to the network. The requisite configuration is automatic; it is a serverless mechanism.

- Security: IPv6 includes security in its specifications such as payload encryption and authentication of the source of the communication. End-to-end security, with built-in strong IP-layer encryption and authentication (embedded security support with mandatory IP Security (IPsec) implementation) is supported.

In MIPv6 each MN is always identified by its "home address," ${ }^{3}$ regardless of its current point of attachment to the Internet; namely, an $\mathrm{MN}$ is always expected to be addressable at its home address whether it is currently attached to its home link or is away from home. The home address is an IPv6 address assigned to the MN within its home subnet prefix on its home link. While an $\mathrm{MN}$ is at home, packets addressed to its home address are routed to the MN's home link [2].

While situated away from its home, an $\mathrm{MN}$ is also associated with an "in-care of" address (CoA) that provides information about the MN's current location. IPv6 packets addressed to an MN's home address are transparently routed to its CoA. A CoA is an IPv6 address associated with an MN that has a subnet prefix from a particular foreign link. The protocol enables IPv6 nodes to cache the binding of an MN's home address with its CoA, and then to send any packets destined for the

\footnotetext{
${ }^{3}$ The acronym HoA is used by some; we do not.
} 
TABLE 1.1 Basic MIPv6 Glossary

\begin{tabular}{|c|c|}
\hline Concept & Definition \\
\hline Care-of address (CoA) & $\begin{array}{l}\text { IPv6 address of mobile node (MN) at its current Internet } \\
\text { attachment point. }\end{array}$ \\
\hline Correspondent node $(\mathrm{CN})$ & $\begin{array}{l}\text { An IPv6 device that is communicating with a mobile node } \\
\text { (MN in MIPv6) or a mobile network node (MNN in } \\
\text { NEMO), using MIPv6 techniques. }\end{array}$ \\
\hline Home agent (HA) & $\begin{array}{l}\text { Host, specifically a router, on the Home Network that } \\
\text { enables the mobile node (MN in MIPv6) or the mobile } \\
\text { network node (MNN in NEMO) to roam (i.e., being "away } \\
\text { from home"). }\end{array}$ \\
\hline Home network (HN) & $\begin{array}{l}\text { The network that a mobile node (MN in MIPv6) or mobile } \\
\text { network node (MNN in NEMO) belongs to when it is not } \\
\text { roaming (when it is at home). That is, the network that is } \\
\text { associated with the network link of the HA. }\end{array}$ \\
\hline Mobile IPv6 (MIPv6) & $\begin{array}{l}\text { An IP protocol, specifically utilizing IPv6 and described in } \\
\text { RFC } 3775 \text { that offers direct communication between MNs } \\
\text { and far-end destinations while retaining the same explicit } \\
\text { IP address regardless of the real-time location or specific } \\
\text { network elements and/or networks used to support } \\
\text { connectivity. }\end{array}$ \\
\hline $\begin{array}{l}\text { Mobile network node } \\
(\mathrm{MNN})\end{array}$ & $\begin{array}{l}\text { (NEMO concept.) Any IPv6 device on a mobile network. } \\
\text { MNNs may be permanently associated (connected) to the } \\
\text { mobile network or visiting the mobile network as } \\
\text { mobile/roaming nodes; they do not need to be aware of the } \\
\text { network's mobility. }\end{array}$ \\
\hline Mobile node (MN) & $\begin{array}{l}\text { An IPv6 device capable of changing its attachment point to } \\
\text { the Internet while maintaining higher layer connectivity } \\
\text { through mobility functionality. }\end{array}$ \\
\hline Mobile router (MR) & $\begin{array}{l}\text { (NEMO concept.) A router capable of changing its point of } \\
\text { attachment to the Internet without disrupting higher layer } \\
\text { connections of attached devices. }\end{array}$ \\
\hline $\begin{array}{l}\text { Network mobility (NEMO) } \\
\text { basic support protocol }\end{array}$ & $\begin{array}{l}\text { An extension to MIPv6 described in RFC } 3963 \text { that enables } \\
\text { mobile network nodes (MNNs) to attach to different points } \\
\text { in the Internet while allowing session continuity for every } \\
\text { node in the mobile network, even as the network moves. }\end{array}$ \\
\hline
\end{tabular}

MN directly to it at this CoA. To support this operation, MIPv6 defines a new IPv6 protocol and a new destination option. All IPv6 nodes, whether mobile or stationary, can communicate with MNs. Table 1.1 provides a basic glossary of key MIPv6 terms; refer to the Glossary for a more extensive list.

The basic apparatus MIPv6 is as follows. Communications must be maintained (e.g., TCP sessions) while the MN is physically moving and is "away from home." To do this, a globally unique, explicit IPv6 address, the home address just discussed, is assigned to every $\mathrm{MN}$; an $\mathrm{MN}$ is always reachable on its home address. This address 
enables the MN to be identified by the far-end node, known as correspondent node $(\mathrm{CN})$. Besides the HA, any other node communicating with an $\mathrm{MN}$ is referred to as a CN. While away from home, an MN obtains the CoA that is typically provided via autoconfiguration. Summarizing this discussion, IPv6-based mobility makes use of two IP addresses per mobile host:

1. One permanent IP address (also known, as noted, as the "home address") is used for identification

2. Another IP address that changes depending on the current location the MN (called, as noted, the care-of address or $\mathrm{CoA}$ ) is used for routing.

The association between an MN's home address and the CoA is known as a "binding" for the MN. While away from home, an MN registers its primary CoA with a well-known location, a router on its home link, thus requesting this router to function as the HA for the MN. The MN performs this binding registration by sending a "binding update (BU)" message to the HA (see Figure 1.2). The HA is and/or acts as a router, a device that forwards IP packets not explicitly addressed to itself; hence, the HA is used to support connectivity on the "upstream link" (MN to $\mathrm{CN}$ ). On the "downstream link" (CN to $\mathrm{MN}$ ), the $\mathrm{CN}$ performs packet routing toward the $\mathrm{MN}$ using the routing header. The $\mathrm{CN}$ learns the position of an $\mathrm{MN}$ by processing BUs. The CN is able to "put" and/or "get" BUs in or from the binding cache. The MN receives router advertisements that specify the prefix of the visited remote locations. The MN then appends the prefix to its interface ID.

The HA (a router in the MN's home network) supports the following functions (also see Figure 1.3):

- Intercept packets that arrive at the MN's home network and whose destination address is its HA

- Tunnel (i.e., provide IPv6 encapsulation) these packets to the MN

- Provide reverse tunneling from the $\mathrm{MN}$ to the $\mathrm{CN}$

MNs can provide information about their current location to CNs, again using BUs and binding acknowledgments (BAs). In addition, return-routability test is performed between the MN, HA, and the $\mathrm{CN}$ in order to authorize the establishment of the binding. Packets between the MN and the $\mathrm{CN}$ are either tunneled via the HA, or sent directly if a binding exists in the $\mathrm{CN}$ for the current location of the MN. In MIPv6, IPsec is specified as the means of securing signaling messages between the MN and HA. MIPv6 tunnels payload packets between the MN and the HA in both directions; this tunneling uses IPv6 encapsulation methods; where these tunnels need to be secured, they are replaced by IPsec tunnels.

MIPv6 is a native extension of IPv6; MIPv6 is seen by some as an application that can, in fact, foster the broader deployment of IPv6 [3]. MIPv6 is a mature protocol with several implementations across the industry that have undergone interoperability testing [4]. 
Top: single CoA included in binding cache per RFC 3775

Binding cache database: correspondent node's binding binding [2001:db8: :EUI CoA1]

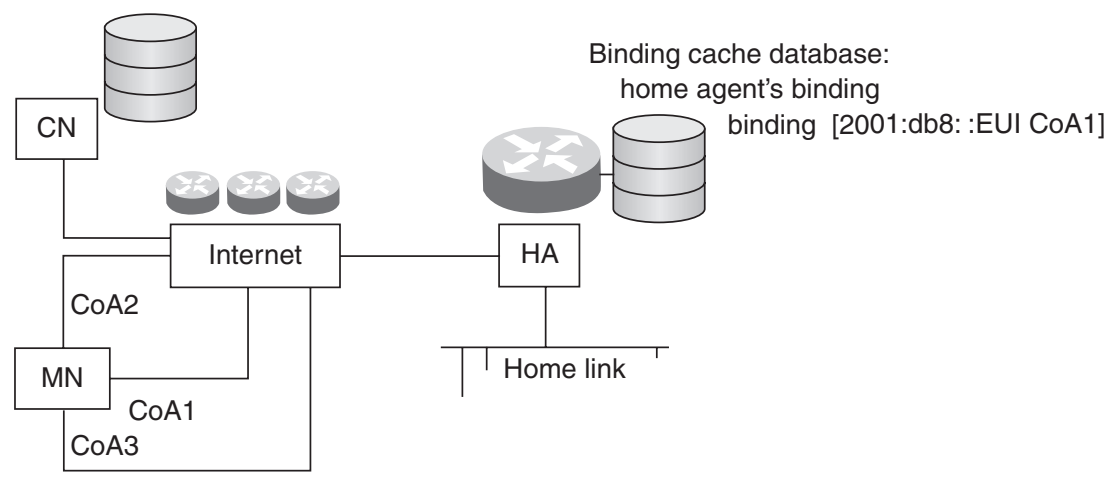

Bottom: multiple CoAs included in binding cache per RFC 5648

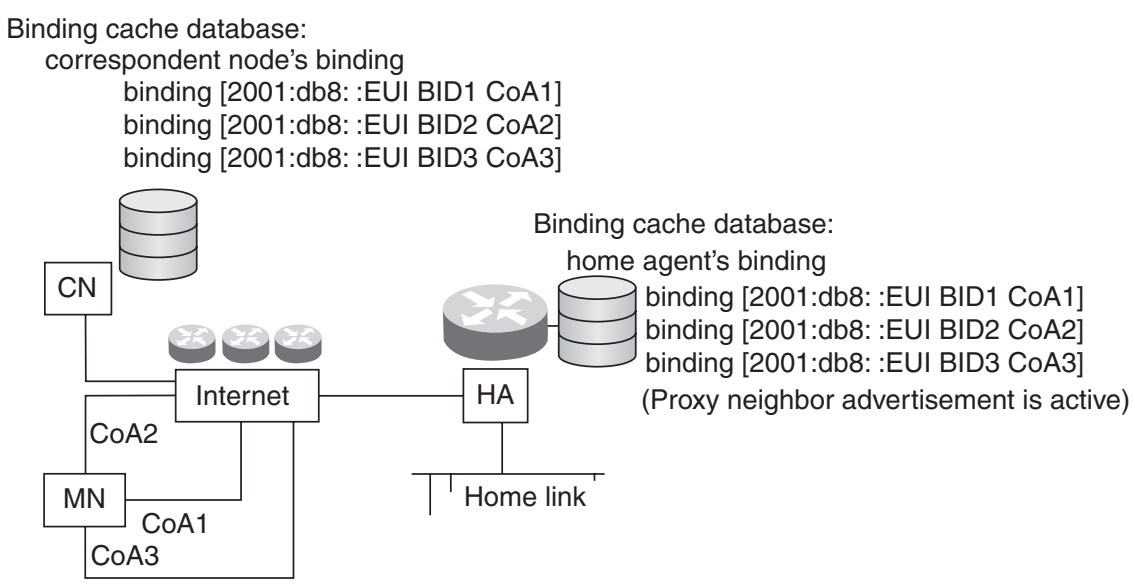

BID: binding identification number

FIGURE 1.2 CoA Registration

MIPv6 makes use of the header structure as defined originally in RFC 2460 and adds a new extension header, specifically a mobility header; this entails a new routing header type and a new destination option. IPv6 extension headers are optional headers that may follow the basic IPv6 header. An IPv6 protocol data unit (PDU) may include zero, one, or multiple extension headers; when multiple extension headers are used, they form a chained list of headers identified by the Next Header field of the previous header. Figure 1.4 depicts the IPv6 packet with some header extensions. The extension header is utilized by the $\mathrm{CN}$, the $\mathrm{MN}$, and the $\mathrm{HA}$ in all the communication transmissions for the bindings. Figure 1.5 depicts the mobility header for MIPv6. 


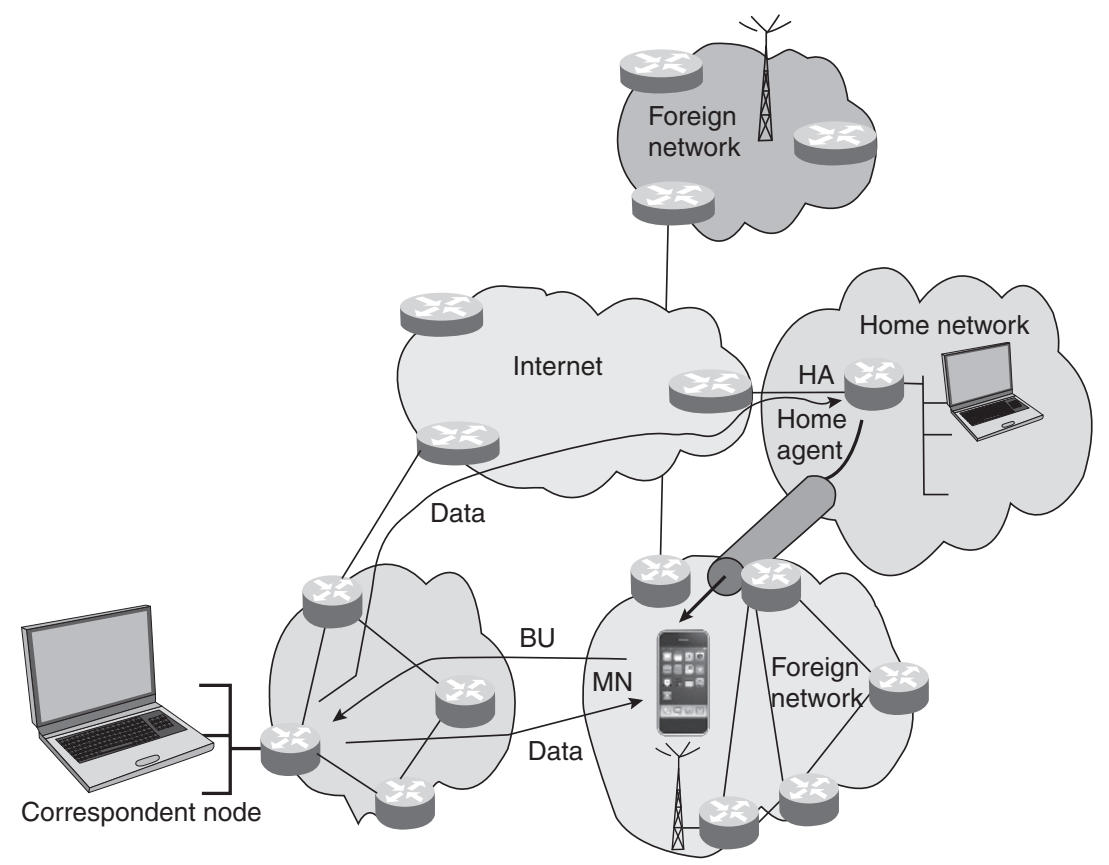

FIGURE 1.3 Communication Supported through the HA

Network mobility (NEMO) basic support protocol as described in RFC 3963 provides additional mobility capabilities. NEMO basic support protocol enables mobile networks (or more specifically mobile network nodes) to attach to different points in the Internet. The protocol is an extension of MIPv6 and allows session continuity for every node in the mobile network as the network moves; it also allows every node in the mobile network to be reachable while moving around. NEMO basic aims at providing continuous Internet connectivity to nodes located in an IPv6 mobile network: an example could be an in-vehicle embedded IP network in a train, bus, airplane, that supports multiple users (say via a WiFi in-vehicle connection). The mobile router (MR), which connects the network to the Internet, runs the NEMO basic support protocol with its HA. The protocol is designed so that network mobility is transparent to the nodes inside the mobile network [5]. Typically, MR implement NEMO functionality for achieving network mobility; however, an MR may also function as an MN. Hence, in summary, NEMO is an extension to the MIPv6 protocol that facilitates the movement of an entire network.

In addition, extensions to MIPv6 and NEMO standards have been offered that allow the registration of IPv4 addresses and prefixes, respectively, and the transport of both IPv4 and IPv6 packets over the tunnel to the HA. These extensions also allow the MN to roam over both IPv6 and IPv4, including the case where network address translation $^{4}$ (NAT) is present on the path between the MN and its HA.

${ }^{4}$ The term network address translator is also used. 
P1: OTA/XYZ P2: ABC

JWBS086-c01 JWBS086-Minoli July 12, $2012 \quad$ 8:12 Printer Name: Yet to Come Trim: 6.125in $\times 9.25$ in

BASIC MIPv6 OPERATION

\begin{tabular}{|c|c|c|c|c|c|}
\hline 0 & Version(4) & Traffic class (8) & Flow label (20 & & $\underline{0}$ \\
\hline 1 & & Payload length (16) & Next header (8) & Hop limit (8) & $\overline{1}$ \\
\hline 2 & \multirow{4}{*}{\multicolumn{4}{|c|}{ Source address (128 bits) }} & $\frac{2}{2}$ \\
\hline 3 & & & & & 3 \\
\hline 4 & & & & & \\
\hline 5 & & & & & $\underline{5}$ \\
\hline 6 & \multirow{4}{*}{\multicolumn{4}{|c|}{ Destination address (128 bits) }} & 6 \\
\hline 7 & & & & & $\overline{7}$ \\
\hline 8 & & & & & 8 \\
\hline 9 & & & & & 9 \\
\hline
\end{tabular}

Hop-by-hop

Ext. Header

Dest. Options

Ext. Header

Routing

Ext. Header

Authentication

Ext. Header

Encapsul. Sec.

Ext. Header

Dest. Options

Ext. Header

FIGURE 1.4 Typical IPv6 Protocol Data Unit

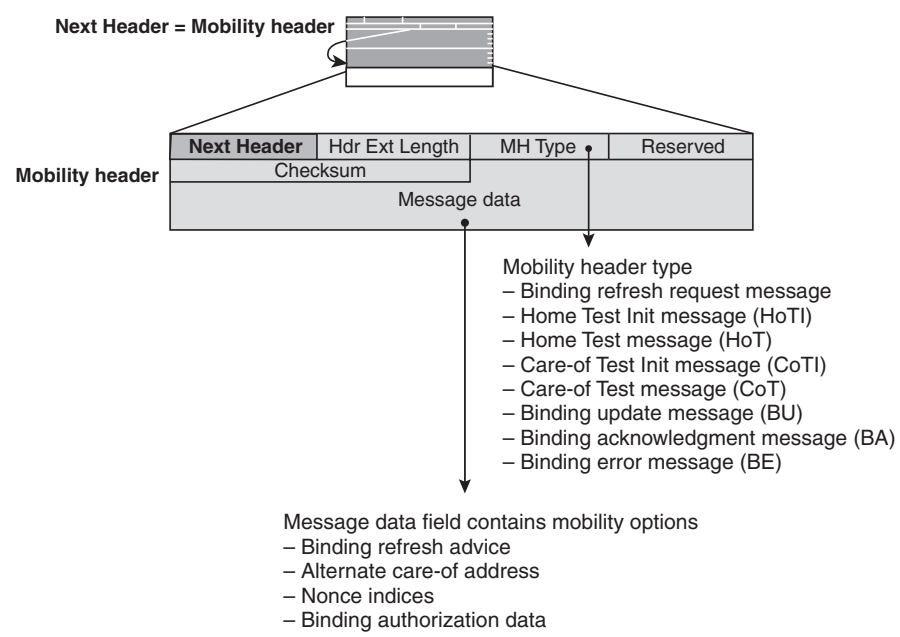

FIGURE 1.5 MIPv6 Mobility Header 
Another extension has also recently been advanced. As we have (implicitly) noted, MIPv6 requires client functionality in the IPv6 stack of an MN. Exchange of signaling messages between the MN and HA enables the creation and maintenance of a binding between the MN's home address and its CoA. Mobility as specified MIPv6 requires the IP host to send IP mobility management signaling messages to the HA, which is located in the network. Network-based mobility is another approach to solving the IP mobility challenge. It is possible to support mobility for IPv6 nodes without host involvement by extending MIPv6 signaling messages between a network node and an HA. This approach to supporting mobility does not require the MN to be involved in the exchange of signaling messages between itself and the HA. A proxy mobility agent in the network performs the signaling with the HA and does the mobility management on behalf of the MN attached to the network. Because of the use and extension of MIPv6 signaling and HA functionality, this protocol is referred to as Proxy Mobile IPv6 (PMIPv6), defined originally in RFC 5213. Network deployments that are designed to support mobility would be agnostic to the capability in the IPv6 stack of the nodes that it serves. IP mobility for nodes that have mobile IP client functionality in the IPv6 stack as well as those nodes that do not, would be supported by enabling PMIPv6 protocol functionality in the network [6].

These various mobility capabilities will be explored in this text.

\subsection{ENTERTAINMENT VIDEO TRENDS}

Mobile video is one of a number of forces that are expected to reshape the video distribution and consumption environments during this decade. Besides mobile video, major other drivers for this evolution include (i) new viewing habits such as time shifting for nonlinear and on-demand content consumption, (ii) new distribution channels (effectively, new content providers, especially Internet based, along with new transport mechanism such as streaming,, (iii) new technologies, and, (iv) standardization of IP-based delivery, especially in conjunction multicast-based IPTV networks and/or with web-based content downloading and social networks.

New viewer paradigms are evolving related to consumption of entertainment video and TV programming that can be summarized as "anywhere, anything, anytime, any platform"; namely, "from any source, any content, in any (encoded) form, at any time, on any user-chosen device, consumed at any location." Mobile video is an example of the new paradigm. Game-changing shifts are also seen on the home front. For example, many new stationary TV sets on the market that now have Ethernet networking connections built directly into the set and require no additional equipment or set-top boxes (STBs) for accessing the Internet; also, many high-end TVs already come with the ability to conduct video calls. In the view of some industry observers these viewer habits, technologies, and approaches will play a part in eventually, perhaps, supplanting broadcast and cable television with Internet programming and distribution. We previously referred to this new paradigm as nontraditional TV (NTTV); new viewer approaches include, but are not limited to the following [7]:

- Watching entertainment/news using the Internet (such as a TV show, a movie, or a short clip) 
- Watching a multicast (rather than broadcast) entertainment/news program

- Watching a VoD program (such as a movie or pay-per-view event) (VoD is also known as content on demand $(\mathrm{CoD}))$

- Watching time-shifted TV (TSTV)

- Utilizing home-based hardware, or

- Utilizing network-based hardware

- Watching entertainment/news with a mobile smartphone, a PDA (personal digital assistant), a videogame console (e.g., the Microsoft Xbox 360 and Sony PlayStation 3), a tablet screen (e.g., Amazon Kindle Fire/Apple iPad/B\&N Nook), or a device in a car or in a yacht

- Watching user-generated content (UGC), particularly utilizing social networks

Some basic service definitions follow:

- Internet television (also known as Internet TV, Online TV) is a television service distributed via the Internet by streaming, as exemplified by services such as Hulu (mostly but not exclusively for US content) and BBC iPlayer (mostly but not exclusively for UK content). The content is typically commercially produced TV material, but the "transmission/distribution" channel is the Internet; the "transmission/distribution" also includes network-resident storage (supported by video servers). Internet TV content is delivered over the open Internet as the term implies (not over a dedicated IP network). Content providers can reach consumers directly, regardless of the carrier or carriers providing the Internet backbone connectivity or Internet access. Video content is accessible from any Internet-ready computer device and is accessible around the world-a consumer does use STBs, although, as noted, increasingly TV sets and STBs have direct Internet connections themselves. Video content is now increasingly available on the Internet. In the past, Internet TV has suffered from low quality; this limitation is now being progressively overcome due to greater bandwidth availability in the Internet core and in the consumer's access subnetwork. Some approaches also use peer-to-peer (P2P) protocols.

- Web television (Web TV, also known as web video) is a genre of digital entertainment distinct from traditional television: in Web TV the content is created specifically for first viewing on the Internet (via broadband access and/or on mobile networks). Web television shows, or Web series, are original episodic shorts (2-9 min per episode at press time, although longer episodes may appear in the future). Web television networks included the following at press time (however, some of these also post TV-originated material): the WB.com ${ }^{5}$, MySpace, YouTube, Blip.tv, HuLu, and Crackle.

- Time-shifted TV. A service or capability that allows the consumer to watch a TV program originally as a broadcast, cable, satellite, or IPTV transmission, that

${ }^{5}$ Companies named in this text are simply illustrative examples of entities that may offer technologies and services under discussion at point in the text; named companies are generally not the only suppliers that may provide such services, and mention of a company and/or service does not imply that such entities or capabilities are recommended herewith, or considered in any way better than others. 
has been time shifted. The time shift service has two flavors. In a basic flavor, the user can preplan the recording of a scheduled TV program (using a local user-owned device, a local cable-provided device, or a remote network-based device - this approach being ideal for mobile environments); the user can watch the program any time later, while still being able to pause, rewind, and resume the playout. Some systems allow the user to skip commercial advertisements during playback. In a more advanced flavor, the service allows a user to halt a scheduled content service in real time and allows the user to continue watching the program later, by providing buffering for pause, rewind, and resume functions. Some refer to TSTV as "catch-up TV," being that it allows consumers to watch a broadcaster's program at their own convenience. Hence, TSTV implies the capture of (what was) a live TV program, either by a customer device or a userprogrammable network-resident device, for playback within a relatively short time (up to a few days). Time shifting does not include, in our definition, VoD downloads of a commercially packaged video clip from a cable TV provider or from an Internet site.

- IPTV is a framework and architecture that, when instantiated in an actual network, supports efficient distribution of (targeted) multimedia services such as television/video/audio/text/graphics/data. The content is delivered over IP-based networks (these being IPv4-based and/or IPv6-based, instead of being traditional cable based) that are tightly managed to support the required level of quality of service/quality of experience (QoS/QoE), security, interactivity, and reliability. Its services are provided to customers via a subscription mechanism very similar to traditional cable TV service.

Collectively we refer to the first two approaches listed above as Internet-based TV (IBTV). Internet-based devices that support IBTV viewing are becoming more popular, ranging for stationary systems from hybrid Internet-ready STBs and digital video recorders (DVRs), to Home theater PCs (HTPCs) (that obviously are Internet ready), to Internet-ready TV sets. An HTPC is a converged device that combines a personal computer with a software application that supports video playback; the HTPC unit is typically colocated with a home entertainment system. On the other hand, new Internet-ready TV sets bypass the PC altogether and access the Internet directly; these sets support the concept of "connected TV (CTV)"; CTVs are also known in some circles as "smart TVs." By the end of 2015, more than 500 million Internet-connected TVs are expected to be in people's homes. Netflix ${ }^{\circledR}$, Amazon, and Apple are (reportedly) "banking" on the idea that the Internet in general, and cloud computing services in particular, are going to be a game changer for home entertainment and that the TV screen can be seen as a "big iPad."

\footnotetext{
${ }^{6}$ The examples of commercial services and service providers identified at various points throughout this text are intended only to depict what we believe to be persistent technical/usage trends. Some of these service, providers, or products may disappear-yet other will emerge. Hence, we believe that the general trends discussed here, as a whole, will persist and prevail.
} 
(Some) operators are already deploying MIPv6 in cellular networks. One example of such networks is CDMA-based networks as defined in the 3GPP2 X.S0011-002-D specification [8]. Mobile WiMAX (worldwide interoperability for microwave access), which is based on IEEE 802.16e, also specifies in the network architecture the use of MIPv6 [9]. Hence, our interest on a text that addresses this topic.

Industry observations such as this one [10] describe some of the important issues that have to be taken into account by stakeholders:

In the last few years, there has been a tremendous increase in mobile video content generation and delivery. This trend has forced the service providers to understand the limitations of the current wired and wireless networks (Internet, WiFi, 3G/4G cellular) and bring new technologies for delivering the video content to the end-user. Last mile access technologies play a crucial role in determining the overall performance and end-user experience of multimodal applications; diverse approaches and technologies (WiMAX, LTE) have recently gained momentum to address this issue. Moreover, offering high quality mobile video services on resource-constrained and heterogeneous mobile devices with varying display sizes, processing powers, network conditions, and battery levels opens up many new challenges. Recent developments such as social media production, multimodal sensing and context technologies, will have an impact on mobile video applications. In fact, supporting a broad spectrum of video-centric applications such as live video streaming, $\mathrm{VoD}$, video games and virtual environments, ...., multimedia conferencing and telepresence, surveillance, sensing and social media on mobile devices demands application-specific techniques that adapt to the underlying network and device architectures.

According to the Cisco Visual Networking Index (VNI) Global Mobile Data Traffic Forecast for 2011 to 2016, worldwide mobile data traffic will increase 18-fold over the next 5 years, reaching 10.8 exabytes per month by 2016 . The increase in mobile traffic is due to a projected surge in the number of mobile Internet-connected devices, which will exceed the number of people on earth, estimated by then at 7.3 billion. During 2011-2016, Cisco anticipates that global mobile data traffic will outgrow global fixed data traffic by three times. The forecast predicts an annual run rate of 130 exabytes of mobile data traffic; this increase represents a compound annual growth rate (CAGR) of 78 percent spanning the forecast period. The following trends are driving these significant increases [11]:

- More streamed content: With the consumer expectations increasingly requiring on-demand or streamed content versus simply downloaded content, mobile cloud traffic will increase, growing 28-fold from 2011 to 2016, a CAGR of 95 percent.

- More mobile connections: There will be more than 10 billion mobile Internetconnected devices in 2016.

- Enhanced computing of devices: Mobile devices are becoming more powerful and thus able to consume and generate more data traffic. Tablets are expected to be generating traffic levels that will grow 62-fold from 2011 to 2016-the 
TABLE 1.2 Bandwidth Trends for Mobile Devices

\begin{tabular}{lrrrrrrr}
\hline & 2011 & 2012 & 2013 & 2014 & 2015 & 2016 & CAGR \\
\hline $\begin{array}{l}\text { Average mobile connection } \\
\quad \text { speed (Kbps) }\end{array}$ & 315 & 504 & 792 & 1,236 & 1,908 & 2,873 & $56 \%$ \\
$\begin{array}{c}\text { Average smartphone } \\
\text { connection speed (Kbps) }\end{array}$ & 1,344 & 1,829 & 2,425 & 3,166 & 4,102 & 5,244 & $31 \%$ \\
\hline
\end{tabular}

highest growth rate of any device category tracked in the forecast. The amount of mobile data traffic generated by tablets in 2016 ( 1 exabyte per month) will be four times the total amount of monthly global mobile data traffic in 2010 (237 petabytes per month).

- Faster mobile speeds: Mobile network connection speed is a key enabler for mobile data traffic growth. More speed means more consumption, and Cisco projects mobile speeds (including $2 \mathrm{G}, 3 \mathrm{G}$ and $4 \mathrm{G}$ networks) to increase ninefold from 2011 to 2016.

- More mobile video: Mobile users want the best experiences they can have and that generally means mobile video, which will comprise 71 percent of all mobile data traffic by 2016.

The Cisco study also projects that 71 percent of all smartphones and tablets (1.6 billion) could be capable of connecting to an IPv6 mobile network by 2016. From a broader perspective, 39 percent of all global mobile devices (more than 4 billion) are expected to be IPv6-capable by 2016. The following regions are experiencing the greatest growth:

- Middle East and Africa will have the highest regional mobile data traffic growth rate with a CAGR of 104 percent, or 36-fold growth.

- Asia-Pacific will have an 84 percent CAGR, or 21-fold growth.

- Central and Eastern Europe will have an 83 percent CAGR, or 21-fold growth.

- Latin America will have a 79 percent CAGR, or 18-fold growth.

- North America will have a 75 percent CAGR, or 17-fold growth.

- Western Europe will have a 68 percent CAGR, or 14-fold growth.

The average mobile connection speed is expected to increase ninefold by 2016; mobile connection speeds are a key factor in supporting and accommodating mobile data traffic growth (see Table 1.2).

\subsection{SCOPE OF INVESTIGATION}

This book aims at exploring these evolving trends, including mobile video trends. It also aims at and offering practical suggestions of how these technologies can 
be implemented in the service provider networks to support cost-effective delivery of entertainment, especially considering the shifts in viewing habit, and how new revenue-generating services could be brought to the market. Chapter 2 provides some IPv6 basics. Chapter 3 provides an overview of MIPv6 protocols, approaches, and technologies. Chapters 4 and 5 describe some more advanced extensions and capabilities that may be of interest to developers. Chapter 6 looks at the capabilities offered by PMIPv6. Chapter 7 addresses security considerations in MIPv6. Chapter 8 looks at some alternative mobile video technologies and also video streaming services, including 3/4G services; finally it assesses MIPv6 support in video environments along with implementations and future directions.

This is believed to be the first book on MIPv6 with applications to linear and nonlinear video distribution. This work will be of interest to planners, CTOs, and engineers at broadcast TV operations, cable TV operations, satellite operations, Internet and ISP providers, telcos, and wireless providers, both domestically and in the rest of the world. Also, it will be of interest to set top box developers, storage vendors, content developers, content distribution outfits, and content aggregators.

We are not implying in this text that IPv6 and/or MIPv6 is strictly and uniquely required to support IP-based mobile video; we are advocating, however, that that platforms based on these protocols provide an ideal, future-proof, and scalable environment for such evolving content-delivery services.

\section{APPENDIX 1.1A: STATISTICS}

This appendix provides some recent basic background data that supports the assertions made earlier in the chapter about the growing importance of mobile video consumption, as actually measured, not forecast, at press time. The data is summarized from data published by Nielsen Company [12]. Table 1.A1 provides overall usage by population, while Table 1.A2 depicts actual hours of usage by service category.

TABLE 1.A1 Overall Usage-Number of Users 2+ (in 000s)-Monthly Reach

\begin{tabular}{lrrr}
\hline & & & Yr to Yr \\
& Q1,2011 & Q1, 2010 & Difference (\%) \\
\hline Watching TV in the home & 288,500 & 286,225 & 0.8 \\
Watching time-shifted TV (all TV homes) & 107,065 & 94,599 & 13.2 \\
Using the Internet on a computer & 190,913 & 191,301 & -0.2 \\
Watching video on Internet & 142,437 & 135,855 & 4.8 \\
Using a mobile phone & 231,000 & 229,495 & 0.7 \\
Mobile subscribers watching video on a & 28,538 & 20,284 & 41.0 \\
$\quad$ & & & \\
\hline
\end{tabular}


TABLE 1.A2 Monthly Time Spent in H:Min-Per User 2+

\begin{tabular}{lrrrc}
\hline & & $\begin{array}{r}\text { Yr to Yr } \\
\text { Difference } \\
\end{array}$ & $\begin{array}{l}\text { Yr to Yr } \\
\text { Difference } \\
\text { (H:Min) }\end{array}$ \\
\hline Watching TV in the home & $158: 47$ & $158: 25$ & 0.2 & $0: 22$ \\
Watching time-shifted TV (all TV homes) & $10: 46$ & $9: 36$ & 12.2 & $1: 10$ \\
DVR playback (only in homes with DVRs) & $26: 14$ & $25: 48$ & 1.7 & $0: 26$ \\
Using the Internet on a computer & $25: 33$ & $25: 54$ & -1.4 & $-0: 21$ \\
Watching video on Internet & $4: 33$ & $3: 23$ & 34.5 & $1: 10$ \\
Mobile subscribers watching video on a & $4: 20$ & $3: 37$ & 20.0 & $0: 43$ \\
$\quad$ mobile phone & & & & \\
\hline
\end{tabular}

\section{APPENDIX 1.1B: BIBLIOGRAPHY}

There were only three books on IPV6 mobile, although there were around 40 RFCs on the topic as of press time, comprising hundreds of pages of key technical material of possible interest to readers and implementers (without counting the numerous other IPv6 RFCs). These books do not focus on video applications, as we do here. Also, a number of (major) developments have taken place since these books were first written and/or published.

- Qing Li, Tatuya Jinmei, and Keiichi Shima, Mobile IPv6: Protocols and Implementation, Morgan Kaufmann, Google eBook, 2009.

- Rajeev S. Koodli and Charles E. Perkins, Mobile inter-networking with IPv6: Concepts, Principles, and Practices, John Wiley \& Sons, 2007.

- Hesham Soliman, Mobile IPv6: Mobility in a Wireless Internet, Addison-Wesley, 2004.

MIPv6 RFCs are listed below. This text provides a complete summary of all these RFCs.

\begin{tabular}{ll}
\hline IETF RFC & Title/Topic \\
\hline RFC 3775 & $\begin{array}{l}\text { Mobility Support in IPv6, June 2004 (Standards Track). } \\
\text { RFC 3776 }\end{array}$ \\
$\begin{array}{c}\text { Using IPsec to Protect Mobile IPv6 Signaling Between Mobile Nodes and } \\
\text { Home Agents, June 2004 (Standards Track) (updated by RFC 4877). } \\
\text { RFC 3963 } \\
\text { Network Mobility (NEMO) Basic Support Protocol, January 2005 (Standards } \\
\text { Track). }\end{array}$ \\
$\begin{array}{l}\text { Fast Handovers for Mobile IPv6, July 2005. (Obsoleted by RFC 5268). } \\
\text { RFC 414068 }\end{array}$ \\
$\begin{array}{l}\text { Hierarchical Mobile IPv6 Mobility Management (HMIPv6), August 2005 } \\
\text { (Obsoleted by RFC 5380). }\end{array}$ \\
$\begin{array}{l}\text { Mobile IPv6 Fast Handovers for 802.11 Networks, November 2005 } \\
\text { (Informational). }\end{array}$ \\
\hline
\end{tabular}




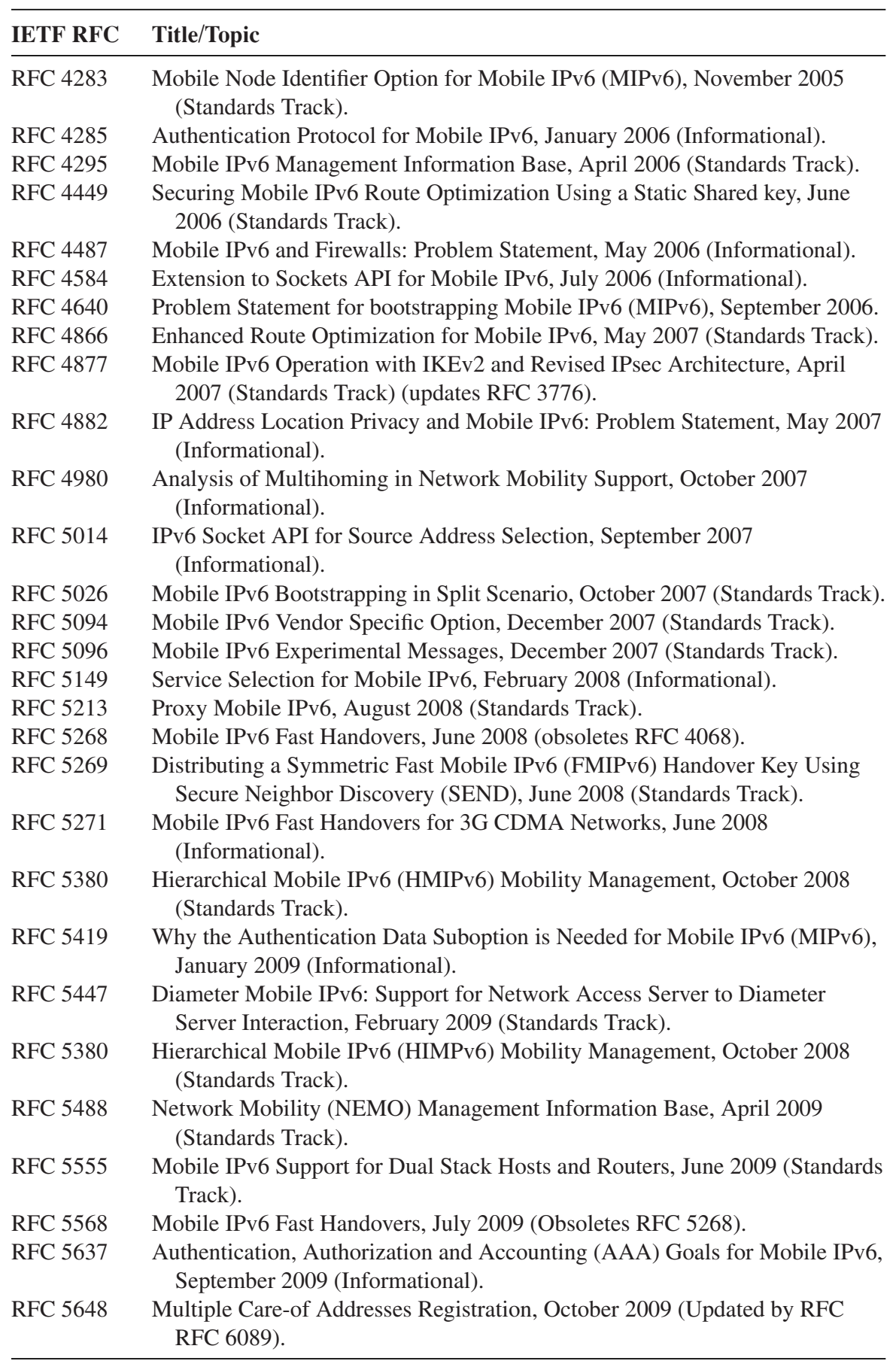




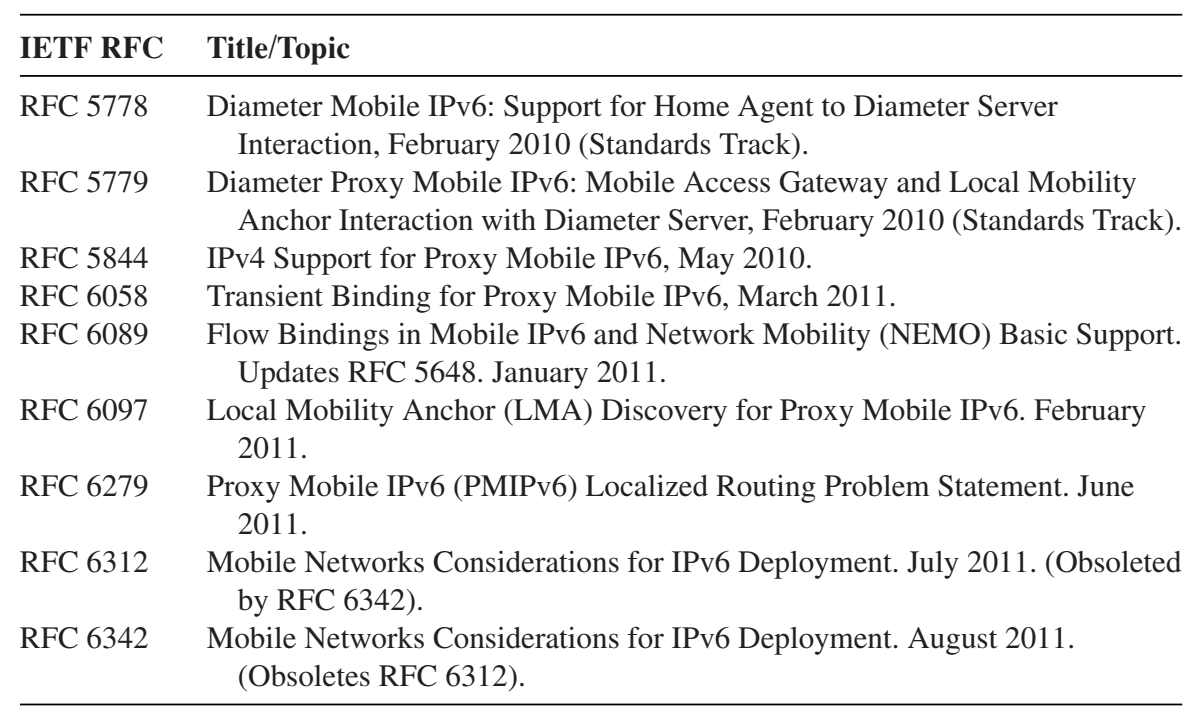

\section{REFERENCES}

[1] D. Levitas, The Mobile DTV Opportunity and Its Role in the Communication Ecosystem, IDC, 2010, 5 Speen Street Framingham, MA 01701, www.idc.com.

[2] J. Arkko, V. Devarapalli, F. Dupont, Using IPsec to Protect Mobile IPv6 Signaling Between Mobile Nodes and Home Agents, RFC 3776, 2004.

[3] 6deploy.org, IPv6 Workshop - IPv6 Mobility Module, 2008.

[4] S. Gundavelli Ed., K. Leung, V. Devarapalli, K. Chowdhury, B. Patil, "Proxy Mobile IPv6", RFC 5213, August 2008.

[5] V. Devarapalli, R. Wakikawa et al, Network Mobility (NEMO) Basic Support Protocol, RFC 3963, 2005.

[6] K. Leung, V. Devarapalli, K. Chowdhury, B. Patil, S. Gundavelli (eds) Proxy Mobile IPv6, RFC 5213, 2008.

[7] D. Minoli, Linear and Non-Linear Video and TV Applications Using IPv6 and IPv6 Multicast, Wiley, 2012. New York, NY.

[8] 3GPP2 X.S0011-002-D, cdma2000 Wireless IP Network Standard: Simple IP and Mobile IP Access Services, http://www.3gpp2.org/Public_html/specs/X.S0011-002-D_v1.0_ 060301.pdf, 2006.

[9] WiMAX Network Architecture - WiMAX End-to-End Network Systems Architecture, 2008, <http://www.wimaxforum.org/documents/documents/WiMAX_Forum_Network_ Architecture_Stage_23_Rel_1v1.2.zip >.

[10] MoVid 2012, Call for Papers (CFP) for 2012 Conference, 2011.

[11] Cisco $^{\circledR}$, Cisco Visual Networking Index (VNI) Global Mobile Data Traffic Forecast for 2011 to 2016, 2012, Cisco Systems, Inc., San Jose, CA.

[12] Nielsen Company, The Cross-Platform Report Quarter 1, 2011, www.nielsen.com. 